\title{
OPINIONES
}

\section{Programa de propagación clonal para Eucalyptus globulus en Chile}

\author{
Clonal Propagation Program for Eucalyptus globulus in Chile \\ C.D.O.: 165.3 \\ ROGER ARNOLD, JUAN CARLOS CARMONA y CLAUDIO BALOCCHI \\ Instituto de Silvicultura, Universidad Austral de Chile, Casilla 567, Valdivia, Chile
}

SUMMARY

Eucalyptus globulus plantations are rapidly expanding in Chile, many pulp manufacturers are now keen to utilize up to $20 \%$ short fibered eucalypt timber in combination with long fibered radiata pine. Variability in unimproved E. globulus seedling plantations combined with heritability data reveal significant potential for genetic improvement in growth, form, and wood quality traits of this species. The genetic improvement program proposed by the 'Cooperativa de Mejoramiento Genético Universidad Austral de Chile-Corporación Nacional Forestal-Empresas Forestales' involves long term recurrent breedings with an initial concentration of resources on developing efficient clonal propagation systems. Clonal rooted cutting will enable optimization of gains from the breeding; a $40 \%$ increase in volume yield is estimated for clonal plantations compared to $20 \%$ for open pollinated seed orchard seedlings. Substantial gains in other traits, including stem for and wood quality are also considered by the program.

\section{RESUMEN}

Las plantaciones comerciales de Eucalyptus globulus en Chile se están expandiendo rápidamente; muchas empresas forestales ligadas a la industria de pulpa y papel están considerando una producción de hasta un $20 \%$ con especies de fibra corta, como lo es Eucalyptus globulus, con la finalidad de combinar esta producción con especies de fibra larga como Pinus radiata. La variabilidad presente en las plantaciones comerciales de E. globulus, combinado con los valores de heredabilidad para las características de interés, revelan el gran potencial que existe para el mejoramiento genético en volumen, forma y propiedades de la madera de esta especie. El programa de mejoramiento genético para eucaliptos propuesto por la Cooperativa de Mejoramiento Genético UACH-CONAF-Empresas Forestales incluye un programa de mejoramiento a largo plazo (Droppelmann y Delmastro, 1988). El programa de la Cooperativa está cimentado en un proceso de selección recurrente con una fase de producción basada en propagación por semillas a través del establecimiento de huertos semilleros. En este programa también se incluye el desarrollo de técnicas de propagación clonal como una alternativa para la reproducción comercial del material mejorado. El desarrollo de técnicas de propagación clonal permitiría optimizar las ganancias esperadas en el programa de mejoramiento genético. Por medio de la propagación clonal masiva del material seleccionado se espera una ganancia en volumen de aproximadamente $40 \%$, comparada con $20 \%$ esperado para el huerto de polinización abierta de primera generación. Substanciales ganancias también se esperan en características como rectitud y propiedades de la madera. Las diferencias en ganancia esperada entre estos dos tipos de propagación van disminuyendo a largo plazo, a medida que se incorporan los resultados de cruzamientos controlados y con ello la propagación de los mejores cruzamientos específicos.

\section{INTRODUCCION}

Las plantaciones de eucaliptos están aumentando significativamente su participación en Chile. Al inicio de 1989 existían más de 70.000 hectáreas de plantaciones de eucaliptos en Chile y los planes de forestación futuros son de aumentar esta superficie a aproximadamente 300.000 hectáreas para el año 2000 (Anónimo, 1990). Muchas empresas de pulpa, que tradicionalmente han usado solamente 
fibra larga de Pinus radiata, actualmente están dispuestas a reemplazar hasta un $20 \%$ de su consumo de madera con fibra corta de eucalipto.

Eucalyptus globulus actualmente es la especie latifoliada exótica de mayor importancia en Chile, su fibra corta permite que su madera tenga una multitud de utilizaciones tanto como fibra o madera sólida. Los papeles manufacturados en base a sus fibras tienen muy buenas características de terminación, densidad, opacidad y una excelente calidad en la formación de hojas (Dean et al., 1990).

Las actuales plantaciones de eucaliptos han sido establecidas con semilla comercial de diferentes fuentes y no presentan ningún grado de mejoramiento genético. Estas plantaciones presentan una gran variabilidad a nivel de árboles, tanto en vigor como en forma. Esta variabilidad poblacional, sumada a la positiva respuesta que presentan las especies de eucaliptos a la manipulación genética, muestran el gran potencial existente para mejorar el crecimiento y las propiedades de la madera a través de selección y cruzamientos. En otros países, ganancias de hasta $100 \%$ o más han sido obtenidas en crecimiento de plantaciones a través del mejoramiento genético (Burley e Ikemori, 1988; Delwaulle et al., 1980).

Con la finalidad de maximizar el rendimiento de Eucalyptus globulus en Chile, la Cooperativa de Mejoramiento Genético UACH-CONAF-Empresas Forestales recientemente formuló un programa de mejoramiento genético para esta especie. Un elemento importante de este programa es el desarrollo de una estrategia dinámica de propagación clonal, esta estrategia se describe en esta publicación.

\section{PROGRAMA DE MEJORAMIENTO GENETICO Y OPCIONES DE PROPAGACION}

El programa de mejoramiento de la Cooperativa, en su fase inicial, considera la selección de árboles "plus" (árboles fenotípicamente superiores seleccionados para ser incluidos en un programa de mejoramiento genético) en plantaciones comerciales. Estas selecciones deben ser probadas en ensayos genéticos y seleccionadas para ser usadas en el mejoramiento recurrente. Existen dos alternativas de propagación del material genéticamente superior para el establecimiento de plantaciones comerciales. La primera alternativa considera el establecimiento de huertos semilleros clonales de polinización abierta o reproducción sexual; la segunda alternativa considera el desarrollo y uso de técnicas de propagación clonal o reproducción asexual (figura 1).

Las ganancias obtenidas en las plantaciones comerciales están en gran medida determinadas por los métodos de propagación utilizados para la multiplicación masiva del material genéticamente superior. El establecimiento de huertos semilleros de polinización abierta permite obtener un nivel básico de ganancia genética. Sin embargo, la propagación vegetativa de genotipos superiores, seleccionados y probados a través de ensayos de progenie, puede proporcionar importantes ganancias adicionales comparadas con las obtenidas en un huerto semillero de polinización abierta. Para E. globulus en Chile la propagación vegetativa a través de estacas ha sido identificada como la estrategia de mayor potencialidad para maximizar las ganancias; esto es en la medida en que se pueda desarrollar una técnica eficiente para la pro-

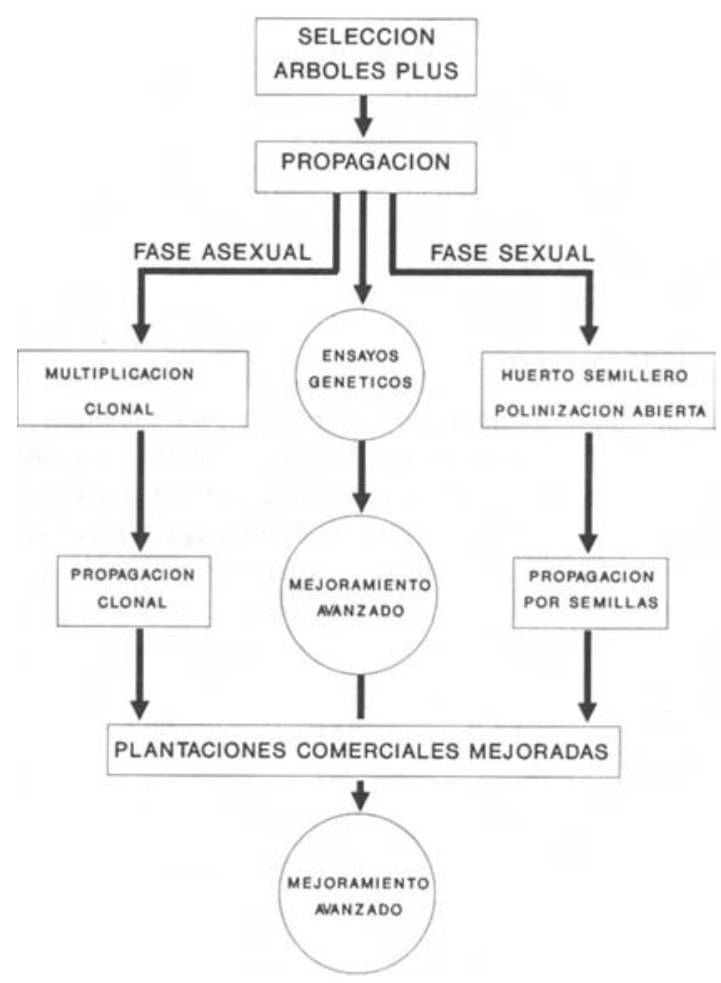

Fig. 1. Fases principales de la estrategia de mejoramiento genético de Eucalyptus globulus en Chile.

Principal stages of the genetic improvement strategy for Eucalyptus globulus in Chile. 
pagación operacional del material seleccionado. En el caso de que la propagación clonal no pueda ser desarrollada operacionalmente, pueden usarse otras alternativas de propagación sexual para aumentar la ganancia genética. Por ejemplo, a través de la propagación a gran escala de familias de hermanos completos.

\section{OBJETIVOS DEL PROGRAMA}

Los objetivos principales del programa de propagación clonal en eucalipto son:

1. Aumentar el actual rendimiento de las plantaciones de E. globulus a través de la incorporación de la propagación clonal como técnica de reproducción del material genéticamente superior.

2. Lograr aumentos adicionales de rendimiento en el largo plazo, como consecuencia de la propagación clonal de nuevos genotipos obtenidos en los programas de mejoramiento avanzado y desarrollo de híbridos.

Como se mencionó anteriormente, el programa inicialmente se concentra en desarrollar sistemas eficientes de propagación de estacas y selección de árboles "plus". Incorpora una programación de actividades que permitiría el inicio de plantaciones clónales mejoradas dentro de 8 años.

\section{DETALLES DEL PROGRAMA CLONAL}

4.1. Selección de árboles "plus". Para iniciar el programa se deben seleccionar árboles superiores en plantaciones "no mejoradas" originadas a partir de semilla. En plantaciones de buena calidad se espera una intensidad de selección de 1 en 1.500 a 1 en 2.000 , con metas iniciales de selección de 1.200 árboles superiores por zona geográfica (la zona geográfica incluye el área de distribución del patrimonio de la empresa).

La selección de estos clones estará basada en las características de forma y crecimiento, incluyendo:

1. Altura

2. Diámetro

3. Rectitud

4. Forma del fuste, se desean fustes cilíndricos

5. Poda natural
6. Diámetro de ramas

7. Ausencia de crecimiento en espiral

8. Copa vigorosa y simétrica

9. Ausencia de pestes y enfermedades.

4.2. Reselección y números de clones. La fase de selección en plantaciones, en base a crecimiento y forma, es la más importante para la identificación de genotipos superiores. Reselecciones posteriores se llevarán a cabo en base a:

1. Capacidad de rebrote de tocón; 2. Capacidad de enraizamiento; 3. Desarrollo en ensayos clonales.

El propósito del proyecto es desarrollar aproximadamente 100 clones por zona a partir de las 1.200 selecciones iniciales (cuadro 1 ).

4.3. Repropagación inicial de los árboles seleccionados. Cada uno de los árboles seleccionados será cortado a una altura de tocón no mayor a los $30 \mathrm{~cm}$ para inducir rebrotes de tocón. Los árboles y la vegetación en un radio de $7 \mathrm{~m}$ de árbol seleccionado serán eliminados para asegurar adecuada luz y con ello un buen desarrollo de los rebrotes. Muestras de madera se obtendrán de los árboles seleccionados para su respectivo análisis y posterior utilización en el proceso de selección clonal.

Los clones que produzcan abundantes rebrotes serán seleccionados para ser repropagados, aquellos que no rebroten adecuadamente se eliminarán del programa clonal. Esta es la primera etapa de reselección clonal.

Cuando presenten un desarrollo adecuado, los rebrotes de tocón serán cosechados para su propa-

\section{CUADRO 1}

Número de genotipos esperados para cada fase de selección del programa de propagación clonal de Eucalyptus globulus.

Numbers of expected genotypes at the different selection stages for the clonal phase of the Eucalyptus globulus tree improvement program

\begin{tabular}{|lc|}
\hline Fase de selección & $\begin{array}{c}\text { Número de clones deseados } \\
\text { (mínimo por zona) }\end{array}$ \\
\hline $\begin{array}{l}\text { 1. Selección árboles "plus" en } \\
\text { plantaciones comerciales }\end{array}$ & 1.200 \\
2. Habilidad de rebrote de tocón & 800 \\
$\begin{array}{l}\text { 3. Capacidad de arraigamiento } \\
\text { 4. Desarrollo en terreno en en- } \\
\text { sayos clonales }\end{array}$ & 300 \\
\hline
\end{tabular}


gación a través de arraigamiento de estacas. Los propágulos obtenidos inicialmente serán utilizados para dos finalidades: ensayos clonales para determinar su desarrollo como estacas, y para el establecimiento de áreas de multiplicación clonal.

4.4. Propagación de estacas. El enraizamiento de estacas de E. globulus requiere de invernaderos con ambiente controlado; temperaturas cálidas y humedades altas son esenciales para optimizar tanto la capacidad de enraizamiento como el crecimiento posterior de las plantas (Orme, 1988a; Orme, 1988b). Otros factores críticos para un enraizamiento exitoso incluyen regímenes hormonales, de fungicidas y de nutrición.

La propagación en invernadero requiere que las estacas sean enraizadas en contenedores. Esta desviación del sistema de raíz desnuda, actualmente usado por la mayoría de los productores forestales, ofrece otros beneficios al programa de plantación, que incluye la extensión del período de plantación y la preparación de una planta más adaptada a condiciones de heladas.

El segundo nivel de reselección clonal consiste en usar solamente aquellos clones con capacidades de enraizamiento superiores al 70 por ciento. Este es un criterio necesario para asegurar el éxito operacional del programa.

4.5. Evaluación clonal. Los clones a propagar operacionalmente serán previamente evaluados en ensayos clonales establecidos en sitios representativos de las futuras plantaciones. Todos los clones deben ser probados en terreno para determinar su desarrollo como estacas.

Los ensayos clonales serán establecidos en varios sitios y deberán incorporar lotes controles para permitir una cuantificación de las ganancias esperadas junto con seleccionar los clones superiores. Las primeras evaluaciones de estos ensayos serán a la edad de 4 a 5 años, lo cual corresponde aproximadamente a la mitad de la edad de rotación mínima contemplada en muchas de las plantaciones pulpables de E. globulus en Chile.

Aumentos sustanciales en ganancia genética serán posibles a través de la reselección de clones basada en los ensayos clonales. Esto corresponde a la tercera fase del programa de reselección clonal. Por otro lado, estos ensayos de terreno permitirán la identificación de clones que se adaptan a condiciones específicas de sitio, tales como clones tolerantes a heladas o con características de madera específicas.
4.6. Areas de multiplicación clonal (AMC). Los propágulos iniciales de cada clon serán también usados para el establecimiento de AMC. La posterior repropagación en cascada, que se refiere al uso del material generado en las AMC para ampliar estas áreas de tal forma de aumentar exponencialmente el número de plantas por clon dentro de las AMC, proporcionará un abundante suministro de estacas para cada clon.

El establecimiento de plantaciones clonales operacionales requiere de la producción de un gran número de estacas en las AMC. Las tasas de multiplicación reales y el espaciamiento óptimo de las plantas madres dentro de las AMC se definirán durante las primeras fases del programa de la Cooperativa.

Las AMC, ubicadas cerca de los invernaderos de enraizamiento, serán manejadas para la rápida producción de un gran número de brotes adecuados para el programa de enraizamiento de estacas. Los clones dentro de las AMC se establecerán en hileras o bloques. Cada árbol será periódicamente podado con la finalidad de mantenerlo a baja altura, esto favorece el desarrollo de la capacidad productiva y mantiene la juvenilidad y el vigor. El manejo intensivo de las AMC incluye riego, fertilización, control de malezas y control de plagas y enfermedades.

4.7. Establecimiento de plantaciones clonales. La optimización de los beneficios de las plantaciones clonales de E. globulus en Chile se logrará plantando los clones seleccionados en bloques monoclonales. La diversidad genética en las plantaciones se asegurará limitando el tamaño del bloque a 25 hectáreas y estableciendo las plantaciones en mosaicos de diferentes clones. Se debe utilizar un mínimo de 15 clones por área.

Dentro de las ventajas de las plantaciones clonales sobre las mezclas multiclonales está el manejo más simple, desde el vivero hasta la plantación, una mayor uniformidad dentro de las plantaciones, mayores productividades por hectárea y un control más fácil de plagas y enfermedades (Burley e Ikemori, 1988).

4.8. Programa de mejoramiento avanzado. El programa de mejoramiento genético de largo plazo, que incluye actividades tales como los cruzamientos controlados y selección recurrente, es la base del mejoramiento genético que se puede obtener en el largo plazo. El programa permanente 
de intercruzamiento de individuos superiores es la única manera de proporcionar ganancias continuas y progresivas a largo plazo. Sin este tipo de programa de mejoramiento, basado en cruzamientos controlados, las ganancias iniciales de los programas clonales permanecerían fijas, dado que no se generarían genotipos superiores a través de nuevos cruzamientos. Nuevo material genético puede además ser generado a través de:

1. Desarrollo de híbridos. Existe el potencial para desarrollar híbridos entre E. globulus y otras especies de eucaliptos con el fin de combinar las características deseables de cada una de las especies padres. Por ejemplo, se pueden lograr grandes beneficios al combinar el crecimiento y propiedades de la madera de E. globulus con el vigor y mayor resistencia a las heladas de Eucalyptus nitens.

2. Intercambio de material genético con otros países. Nuevos recursos genéticos pueden obtenerse a través de la cooperación e intercambio de material con programas de mejoramiento de E. globulus en otros países.

\section{DESARROLLO DE TECNOLOGIA}

Se ha publicitado mucho sobre las técnicas de propagación vegetativa y sobre los éxitos logrados por programas clonales en algunos eucaliptos subtropicales. El principal ejemplo es el trabajo reali- zado por Forestal Aracruz, en Brasil, con Eucalyptus grandis (Camphinos e Ikemori, 1983; Zobel et al., 1983). Sin embargo, no existen en Chile programas operacionales de propagación de estacas en eucaliptos, desconociéndose además la tecnología necesaria para propagar E. globulus, una especie de clima templado que requiere de técnicas más complejas que las usadas en especies subtropicales (Orme, 1988a).

El desarrollo de una técnica de propagación de estacas que permita una eficiente multiplicación operacional en E. globulus es una de las primeras prioridades para el programa de mejoramiento genético de la especie. Sin embargo, una exhaustiva evaluación de los resultados obtenidos para esta especie en países como Australia y Portugal debería preceder las fases más aplicadas de ensayos e investigación en Chile. Sin el desarrollo de un sistema eficiente de propagación clonal, los niveles de ganancia esperados en E. globulus estarán limitados a la propagación por semillas de las mejores familias seleccionadas.

\section{GANANCIAS GENETICAS}

La selección inicial de árboles "plus" proporcionará ganancias sustanciales para volumen, forma y calidad. La ganancia en volumen estimada para una edad de 6 años es de aproximadamente $20 \%$ para huertos semilleros clonales y de $40 \%$ para propagación vegetativa (cuadro 2). Las ga-

\section{CUADRO 2}

Parámetros genéticos (Volker et al. 1990) y estimaciones de ganancia para Eucalyptus globulus a los 6 años de edad

Genetic parameters (Volker et al. 1990) and estimated genetic gains for Eucalyptus globulus (age: 6 years)

\begin{tabular}{|c|c|c|c|c|c|c|c|}
\hline \multirow{2}{*}{$\begin{array}{l}\text { Caracte- } \\
\text { rística }\end{array}$} & \multirow{2}{*}{$\begin{array}{l}\text { Promedio } \\
\text { población }\end{array}$} & \multirow{2}{*}{$\begin{array}{c}\text { Desviación } \\
\text { estándar } \\
\text { fenotípica }\end{array}$} & \multicolumn{2}{|c|}{ Heredabilidad (1) } & \multirow{2}{*}{$\begin{array}{l}\text { Intensidad } \\
\text { selección } \\
\text { (2) }\end{array}$} & \multicolumn{2}{|c|}{ Ganancia genética } \\
\hline & & & $\begin{array}{l}\text { sentido } \\
\text { estricto }\end{array}$ & $\begin{array}{l}\text { sentido } \\
\text { amplio }\end{array}$ & & $\begin{array}{c}\text { huerto } \\
\text { semillero }\end{array}$ & $\begin{array}{l}\text { propagación } \\
\text { clonal (3) }\end{array}$ \\
\hline Altura (m) & 19,0 & 1,24 & 0,12 & 0,24 & 3.367 & $33 \%$ & $66 \%$ \\
\hline Diámetro (cm) & 13,1 & 2,65 & 0,24 & 0,48 & 3.367 & $30 \%$ & $60 \%$ \\
\hline Volumen $\left(\mathrm{dm}^{3}\right)$ & 131,0 & 42,39 & 0,19 & 0,38 & 3.367 & $21 \%$ & $42 \%$ \\
\hline Forma fuste (4) & 4,8 & 0,99 & 0,22 & 0,44 & 3.367 & $15 \%$ & $30 \%$ \\
\hline Tamaño ramas (5) & 2,6 & 0,93 & 0,29 & 0,58 & 3.367 & $35 \%$ & $70 \%$ \\
\hline
\end{tabular}

1. Heredabilidades, en el sentido amplio, se estimaron asumiendo que la varianza genética para crecimiento y forma en $E$. globulus es $50 \%$ aditiva y $50 \%$ no-aditiva.

2. Se asumió una intensidad de selección de 1 en 1.000 para la estimación de ganancias.

3. La ganancia del programa clonal asume que el $100 \%$ de los árboles seleccionados se pueden propagar clonalmente (se mantiene intensidad de selección para volumen y forma).

4. Forma del fuste evaluada subjetivamente en una escala de 0 (peor forma) a 6 (mejor forma).

5. Evaluada subjetivamente en una escala de 1 (ramas gruesas) a 5 (ramas delgadas). 
nancias reales estarán influidas por las correlaciones genéticas entre la edad de 6 años y la edad de rotación elegida. Se espera que dichas correlaciones sean altas dado que se contemplan rotaciones cortas para la mayoría de las plantaciones de $E$. globulus en Chile.

La diferencia en ganancias esperadas para crecimiento y forma entre las estrategias de propagación sexual (huerto semillero de polinización abierta) y propagación asexual (multiplicación clonal), permiten destacar el valor de los programas clonales. Esta es la base para enfatizar el desarrollo de técnicas de propagación vegetativa en las primeras fases del programa de mejoramiento genético de la Cooperativa.

Se espera que las ganancias totales en término de rendimiento neto de pulpa por hectárea exceda la magnitud de la ganancia en volumen; esto se debe a los altos valores de heredabilidad que se han estimado para las características de la madera en E. globulus (cuadro 3). Los mayores valores de heredabilidad para estas características indican un gran potencial de ganancias a través de la manipulación genética. En el programa de Forestal Aracruz, en Brasil, se han obtenido ganancias en volumen $\left(\mathrm{m}^{3} / \mathrm{ha}\right)$ de $112 \%$ más un $23 \%$ de ganancia en rendimiento de pulpa por unidad de volumen (rendimiento de celulosa $/ \mathrm{m}^{3}$ de madera), lo que proporciona una ganancia total de $135 \%$ en términos de productividad forestal (toneladas de celulosa/ha/año) (Anónimo, 1984). El mejoramiento en crecimiento y forma contribuirán también a aumentar la calidad de la madera y a mejorar el rendimiento de pulpa. Fustes más rectos y ramas más pequeñas reducen la proporción de madera de

\section{CUADRO 3}

Valores de heredabilidad para volumen y propiedades de la madera en Eucalyptus globulus (Dean et al. 1990)

Heretability values for volume and wood quality traits reported for Eucalyptus globulus (Dean et al., 1990)

\begin{tabular}{|lc|}
\hline Característica & $\begin{array}{c}\text { Heredabilidad } \\
\text { sentido estricto }\end{array}$ \\
\hline Volumen & 0,18 \\
Corteza (\% sobre volumen) & 1,00 \\
Humedad (\%) & 0,82 \\
Densidad de la madera & 0,78 \\
Rendimiento de pulpa $(\%)$ & 0,28 \\
\hline
\end{tabular}

tensión y el contenido de extraíbles, resultando en un aumento neto en el rendimiento de celulosa.

El mayor vigor de los árboles, el mayor rendimiento volumétrico y la mayor uniformidad se traducirán en muchos otros beneficios, tales como:

1. Reducción en las actividades de control de malezas. El crecimiento inicial más rápido de los árboles reduce el período en el cual están afectados en forma adversa por las malezas competitivas.

2. Reducción en los costos de cosecha y transporte. Volúmenes más altos por hectárea combinados con mayores tamaños de trozos aumentan la eficiencia. La mayor uniformidad del rodal puede aumentar la factibilidad de cosecha mecanizada.

3. El mayor crecimiento permitirá el uso de rotaciones más cortas para obtener el mismo rendimiento volumétrico por unidad de superficie.

4. Una silvicultura simplificada. Una mayor uniformidad del bosque permitirá una programación más exacta y eficiente de los tratamientos silviculturales.

El programa básico de mejoramiento genético para largo plazo es absolutamente esencial para obtener ganancias adicionales en el futuro. La segunda generación de mejoramiento bien podría proporcionar ganancias similares o mayores que las esperadas para la primera generación de mejoramiento.

\section{CONCLUSIONES}

Las plantaciones de E. globulus establecidas actualmente en Chile, utilizando semilla no mejorada, revelan el potencial de esta especie para producir altos rendimiento de pulpa de fibra corta de buena calidad. El mejoramiento genético de esta especie proporcionará un mejoramiento significativo, tanto en el rendimiento volumétrico como en las propiedades de la madera de las plantaciones.

El desarrollo de sistemas eficientes de propagación vegetativa es una prioridad para la optimización de las ganancias derivadas del programa de mejoramiento genético. La reselección clonal, en base a características tales como propiedades de la madera y/o adaptabilidad a sitios específicos, permitirá que todas las empresas miembros de la Cooperativa puedan desarrollar el material genético que mejor se adapte a sus condiciones de sitio o producto. 
La naturaleza cooperativa del programa optimizará los retornos a través del desarrollo de un programa de mejoramiento genético dinámico. Empresas individuales lograrán ahorros sustanciales a través del intercambio y uso de clones seleccionados, en la misma zona, por otros miembros de la Cooperativa. Esto reducirá en forma significativa el número de selecciones originales que tendrían que hacer las empresas en forma individual.

\section{BIBLIOGRAFIA}

ANONIMO, 1984. "Brazil's new forest soars to success". Pulp and Paper International. December 1984. 26(12).

ANONIMO, 1990. "Chile launches into eucalyptus". Chilean Forestry News. February-March 1990.

BURLEY, J., IKEMORI, J. 1988. "Tropical forest production: the impact of clonal forest propagation technology". En: Towards an Agro-Industrial Future. Stonleigh,
Kensilwork, Warwickshire, England. Royal Agricultural Society of England. Monograph Series $N^{\circ} 1$ : 169-180.

CAMPHINOS, E., IKEMORI, Y. 1983. "Production of vegetative propagules of eucalyptus species by rooting of cuttings". En: 2nd Symposium on Plantation Forestry in the Neotropics - Its Role as a Source of Energy. IUFRO Group S. 1.07.09. Vicosa, Brasil, 8 pp.

DEAN, G.H., FRENCH, J., TIBBITS, W.N. 1990. "Variation in pulp and paper making characteristics in a field trial of Eucalyptus globulus. En: $44^{\text {th }}$ APPITA, Annual General Conference. Rotorua, New Zealand. April 1990, 12 pp.

DELWAULlE, J.C., LAPLACE, Y., QUILLET, G. 1980. "Mass production of rooted cutting in the People's Republic of the Congo". En: IUFRO Symposium and Workshop on Fast Growing Tree Species. Aguas de São Pedro - São Paulo Brazil. August, 1980, 9 pp.

ORME, R.K. 1988a. "Clonal Forestry with termperate eucalypts". En: 42nd APPITA, Conference. Hobart, Tasmania, Australia. April 1988, $11 \mathrm{pp.}$

ORME, R.K. 1988b. "Genetic Improvement of Eucalyptus globulus". En: A.F.D.I. Conference. Albury, Australia. April 1988, 15 pp.

ZOBEL, B., IKEMORI, Y., CAMPHINOS. E. 1983. "Vegetative propagation in eucalyptus". En: Canadian Tree Breeding Conference. Toronto, Canadá, 11 pp. 